\title{
Prevalence and Risk Factors of Urinary Incontinence in Poorly Educated Female Population
}

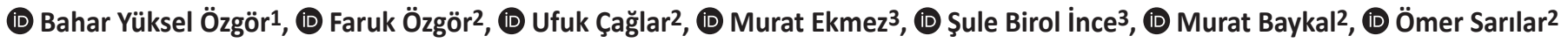 \\ 1Esenler Maternity and Children's Hospital, Clinic of Obstetrics and Gynecology, Istanbul, Turkey \\ 2University of Health Sciences Turkey, Haseki Training and Research Hospital, Clinic of Urology, istanbul, Turkey \\ 3 University of Health Sciences Turkey, Haseki Training and Research Hospital, Clinic of Obstetrics and Gynecology, Istanbul, Turkey
}

\section{Abstract}

Objective: There is a significant inverse correlation between poor education level and one's awareness of his/her health status. Poorly educated patients presume most of the disorders as an expected progress of aging and daily life. In our study we aimed to clarify the prevalence of urinary incontinence (UI) in women with a poor educational level.

Methods: Female patients admitted to urology and gynecology outpatient clinics without UI complaints were enrolled into the study. Patients, who were uneducated or only literate, were defined as poorly educated. All patients were made to fill in Urinary Distress Inventory (UDI-6) and Incontinence Impact Questionnaire (IIQ-7) forms. Patients younger than 18 years, with a history of incontinence surgery and pelvic surgery, history of pelvic radiation, presence of urologic and gynecological malignancy or severe neurological diseases were excluded from the study.

Results: In study population, 236 patients (46.1\%) had a compliant of UI. Patients with UI were at a significantly older age and they had higher Body Mass Index (BMI). UDI-6 and IIQ-7 scores were significantly higher in patients with UI. The BMI, history of difficult labor and presence of pelvic organ prolapse were significantly higher in patients with stress UI (SUI). Age, BMI, presence of diabetes mellitus, number of deliveries, history of difficult labor and presence of pelvic prolapse were significantly higher in patients with urgency UI.

Conclusion: Our study revealed that even not applying to hospitals with that complaints the incidence of UI was very high in poorly educated female population and higher BMI, presence of difficult labor, pelvic prolapse were risk factors for UI and SUI subtypes.

Keywords: Poorly educated, stress, urge incontinence

\section{INTRODUCTION}

Urinary incontinence (UI) is defined as the complaint of any involuntary leakage of urine and forms of UI are divided into three main subtypes as stress UI (SUI), urgency UI (UUI) and mixed UI (MUI), according to International Continence Society (1). Although, $\mathrm{UI}$ is not a life-threatening disorder, its adverse effects on quality of life, mental health and sexual behavior are wellknown. Zaccardi et al. (2) stated that 12\%-50\% of middle-aged women were faced with UI. In another study, Hunskaar et al. (3) stated the wide range of UI prevalence in different countries such as Spain with 23\% and France with almost 45\%. Some authors believed extensive differences between UI rates stem from ethnicity, age at which incontinence begins, heterogeneity of the population studied and educational level.

Previous reports demonstrated the significant inverse correlation between poor education level and one's awareness of his/her health status. Poorly educated patients presuming most of the disorders as an expected progress of aging do not benefit from patient information programs properly, causing inefficient and inadequate description of complaints. Pedro et al. (4) found that infertile people with low education level did not have adequate knowledge about their illness, interventions and campaigns. 
Similarly, Ramírez-Moreno et al. (5) stated low educated patients who were faced with stroke had a lower quality of health and life expectancy than high-educated patients. Also, Kubik et al. (6) investigated incontinence prevalence considering ethnicity and has come to the conclusion that low socioeconomic status decreases awareness of incontinence.

Although previous reports investigated the prevalence of $\mathrm{UI}$ in different ages, ethnicities, regions and socioeconomic status intensively, no study has yet to evaluate the impact of educational level. In present study, we primarily aim to clarify the prevalence of UI in women with a poor educational level.

\section{METHODS}

This study was performed after approval by Haseki Training and Research Hospital Ethics Committee (4532771-432). Form was obtained from the patients. Between February 2018 and August 2018, female patients admitted to urology and gynecology outpatient clinics without UI complaints in two hospitals, were enrolled into the study. Patients, who were uneducated or only literate, were defined as poorly educated and selected as candidates for the study. The charts of the patients were recorded prospectively in the electronic data system. Detailed medical history was obtained from each patient. Patients' age, Body Mass Index (BMI), presence of diabetes mellitus, menopause status, number of deliveries and abortuses were evaluated. Also, patients were made to fill in Urinary Distress Inventory (UDI-6) and Incontinence Impact Questionnaire (IIQ-7) forms. Exclusion criteria were being <18 years old, history of incontinence surgery and pelvic surgery, history of pelvic radiation, presence of urologic and gynecological malignancy. Also, patients with severe neurological diseases and patients who failed to fill in the form were excluded from the study.

\section{Urinary Distress Inventory-6 Questionnaire}

The UDI-6 questionnaire is a simple and lucid form to evaluate quality of life in patients with urinary incontinence. The UDI6 evaluates the type and severity of urological symptoms with 6 questions and each question is scaled from zero to three according to the volume of the complaints. The questionnaire is divided into three subgroups of irritability symptoms, stress symptoms and obstruction symptoms/pain related to urination. Each question was designed with two questions. To obtain UDI-6 score for each patient, sum of scores was divided by six and then, multiplied by twenty-five (7).

\section{Incontinence Impact Questionnaire-7}

The IIQ form is developed by the Continence Program for Women Research Group with 30 questions to assess the effects of
UI in female patients' quality of life. Then, IIQ form was revised from 30 to 7 items for a more efficient and simplified use (IIQ7). In IIQ-7 form, each question was scaled from 0 to 3.0 refers to "not at all"; 1 refers to "slightly"; 2 refers to "moderately"; and 3 means "greatly". To obtain IIQ-7 score, sum of the scores (range between 0-21) is divided by seven and then multiplied by $33.3(100 / 3)$ and the scores are put on a scale from 0 to 100. If $<2$ response is missing, the average score of the questions is taken. If more than 2 items are missing, a total score cannot be calculated (8).

\section{Statistical Analysis}

The Statistical Package of Social Sciences for Windows (SPSS) version 20.0 was used for statistical analysis. The patients were categorized into groups according to presence of urinary incontinence, urge urinary incontinence, SUI and mixed incontinence. Categorical variables were presented as numbers and analyzed with chi-square test. Continuous variables were presented as mean \pm standard deviation and evaluated with independent sample t-test. Correlation analyses were done by Pearson's correlation coefficient. Logistic regression analyses, on the other hand, were used so as to find predictive factors for urinary incontinence. A statistical significance was noted when two tailed $p$ value $<0.05$.

\section{RESULTS}

At the end of the study period, total of 512 patients were evaluated. The mean age and mean BMI were 49.3 years and $27.2 \mathrm{~kg} / \mathrm{m}^{2}$, respectively. Total of 72 patients (14.1\%) had diabetes mellitus and 234 patients (45.7\%) were in postmenopausal status. The mean number of deliveries was 3.1. Patients' demographic hallmarks were summarized in Table 1.

\begin{tabular}{|l|l|}
\hline \multicolumn{2}{|l|}{ Table 1. Demographic characteristics of patients } \\
\hline Number of patient & 512 \\
\hline Age (year)* & $49.3 \pm 13.5$ \\
\hline BMI (kg/m²)* & $27.2 \pm 4.1$ \\
\hline DM & $72(14.1 \%)$ \\
\hline Menopause & $234(45.7 \%)$ \\
\hline Number of deliveries* & $3.1 \pm 2.1$ \\
\hline Vaginal* & $2.7 \pm 2.3$ \\
\hline Cesarean* & $0.4 \pm 0.8$ \\
\hline Abortus number* & $0.6 \pm 1.0$ \\
\hline History of difficult labor & $103(20.1 \%)$ \\
\hline Pelvic prolapsus & $93(18.2 \%)$ \\
\hline *Mean \pm standard deviation, BMI: Body mass index, DM: Diabetes mellitus \\
\hline
\end{tabular}


In study population, 236 patients (46.1\%) (SUI in 51 patients, UUI in 102 patients and MUI in 83 patients) had a compliant of UI. Comparison of patients with and without $\mathrm{UI}$ has revealed that patients with UI were at a significantly older age and they had higher BMI and the higher rate of women were postmenopausal $(p=0.001, p=0.001, p=0.032$ respectively). Furthermore, the number of vaginal deliveries and abortus was higher $(p=0.011$ and $p=0.001$ ). Furthermore, they went through a difficult labor history, diabetes mellitus (DM) and pelvic organ prolapse ( $p=0.005, p=0.006$ and $p=0.001$ respectively). Also, UDI- 6 and IIQ-7 scores were significantly higher in patients with UI (28.6 vs $9.0, p=0.001$ and 37.3 vs $13.3, p=0.001$, respectively). The BMI $\left(28.7 \mathrm{~kg} / \mathrm{m}^{2}\right.$ vs $\left.25.7 \mathrm{~kg} / \mathrm{m}^{2}, \mathrm{p}=0.001\right)$, presence of pelvic organ prolapse (31.4\% vs $10.1 \%, p=0.001$ ) were significantly higher in patients with SUI. In addition; age, BMI, presence of DM, history of difficult labor and presence of pelvic prolapse were significantly higher in patients with UUI ( $p=0.001$ for each parameter). Also, the number of deliveries was significantly higher in patients with UUI $(p=0.027)$. Moreover, patients with MUI were at a significantly older age and they had a higher BMI, DM and higher rate of postmenopausal status $(p=0.001$, $p=0.001, p=0.013$ and $p=0.012$ respectively). Additionally, abortus numbers and pelvic organ prolapse diagnosis were significantly higher in patients with MUI ( $p=0.001$ and $p=0.02)$. Also, UDI-6 and IIQ-7 scores were significantly higher in all UI subgroups ( $p=0.001$ and $p=0.001$ for each subgroups) (Table 2 ).

Multivariable regression analysis revealed that higher BMI, history of difficult labor and pelvic prolapse were associated with $\mathrm{UI}(\mathrm{p}=0.01, p=0.02$ and $p=0.01$ respectively). Moreover, higher $\mathrm{BMI}$ and presence of pelvic organ prolapse increased the risk of SUI 3.78 and 3.9 times, respectively. Presence of DM, history of difficult labor and pelvic organ prolapse were found as the only risk factors for $\mathrm{UUI}$ in multivariable regression analysis $(\mathrm{p}=0.01$ for each parameter). Lastly, higher BMI, abortion history and pelvic prolapse were the risk factors for MUI ( $p=0.01$ for each parameter) (Table 3).

\section{DISCUSSION}

Education level plays an important role for one to discern the abnormal changes in his/her body and consult a physician. Many previous reports in different disciplines have demonstrated that poorly educated people do not seek medical support until disease begins to hinder daily physical activities, disease progression, or emergence of fatal complications. On the other hand, $\mathrm{UI}$ is not fatal disorder but its association with deteriorating the quality of life is well-known. As a result, diagnosing UI in poor educated

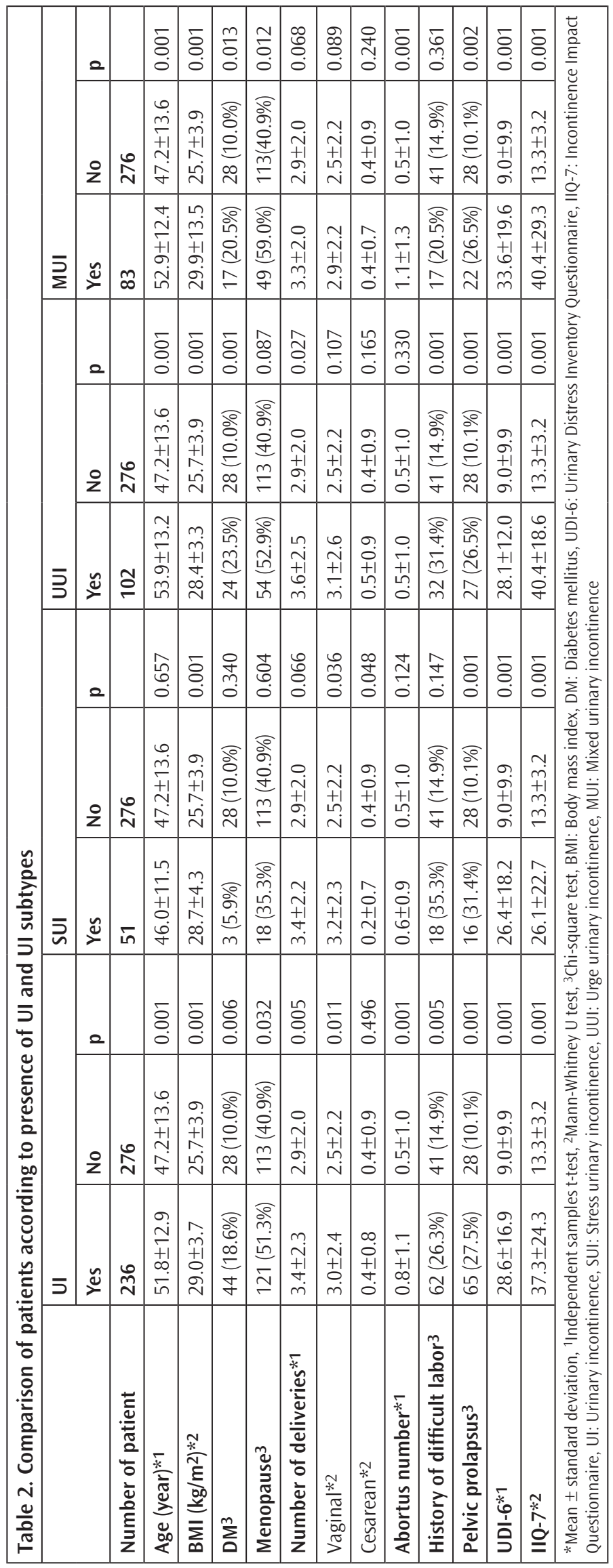


Table 3. Multivariable regression analysis

\begin{tabular}{|c|c|c|c|c|c|c|c|c|}
\hline & \multicolumn{2}{|l|}{ UI } & \multicolumn{2}{|l|}{ SUI } & \multicolumn{2}{|l|}{ UUI } & \multicolumn{2}{|l|}{ MUI } \\
\hline & OR $(95 \% \mathrm{Cl})$ & $\mathrm{p}$ & OR $(95 \% \mathrm{Cl})$ & p & OR $(95 \% \mathrm{Cl})$ & $\mathrm{p}$ & OR $(95 \% \mathrm{CI})$ & p \\
\hline Age* $^{*}$ & $0.97(0.54-1.75)$ & 0.92 & $0.26(0.05-1.33)$ & 0.11 & $1.10(0.53-2.30)$ & 0.80 & $1.36(0.60-3.28)$ & 0.47 \\
\hline DM & $1.34(0.74-2.40)$ & 0.33 & $0.42(0.10-1.78)$ & 0.25 & $1.84(0.91-3.74)$ & 0.09 & $1.12(0.51-2.50)$ & 0.78 \\
\hline Menopause & $1.36(0.64-2.90)$ & 0.42 & $1.42(0.66-3.02)$ & 0.37 & $1.59(0.86-2.94)$ & 0.14 & $1.76(0.90-3.42)$ & 0.10 \\
\hline Abortus & $1.38(0.94-2.15)$ & 0.13 & $1.55(0.76-3.17)$ & 0.23 & $0.76(0.43-1.35)$ & 0.36 & $2.43(1.36-4.35)$ & 0.01 \\
\hline History of difficult labor & $1.89(1.15-3.12)$ & 0.02 & $1.45(0.64-3.27)$ & 0.37 & $2.22(1.22-4.06)$ & 0.01 & $1.55(0.73-3.30)$ & 0.26 \\
\hline Pelvic prolapsus & $3.22(1.88-5.51)$ & 0.01 & $3.90(1.72-8.84)$ & 0.01 & $3.37(1.68-6.73)$ & 0.01 & $3.38(1.51-7.55)$ & 0.01 \\
\hline
\end{tabular}

*Under and over 65 years old, **Under and over 30 years old, ***0, 1, 2, 3 years and older, BMI: Body mass index, DM: Diabetes mellitus, UI: Urinary incontinence, SUI: Stress urinary incontinence, UUI: Urge urinary incontinence, MUI: Mixed urinary incontinence

women contributes to the resolution of a health problem which has been ignored so far.

The effect of advancing age on incontinence is still under investigation. Stephan and Hajjar (9) claimed that UI was an under-diagnosed and under-treated disorder with deteriorated quality of life in elderly population. In another study, Othman et al. (10) were faced with $24.4 \% \mathrm{UI}$ rate in women with $25-34$ years and $32.3 \% \mathrm{UI}$ rate in women with 55-64 years. Also, they reported that only aging was a risk factor in the development of UUI and MUI, not SUI. Similarly, it was found that age was significantly older in patients both with $\mathrm{UI}$ and $\mathrm{UUI}$ and MUI subtypes in univariate analysis. However, multivariable regression analysis revealed that age was not a risk factor in the development of SUI, UUI and MUI in the present study. The mean age was 49.3 years in our study which was a younger average than the average of other studies and that may have had a role on the outcome we came up with.

In obese patients, patients with chronic insulin resistance, increased abdominal pressure and oxidative stress may have a role in the development of UI. Mishra et al. (11) evaluated the relation between obesity and $\mathrm{UI}$ and they concluded that higher BMI was associated with SUI and severe UI in middle aged female patients. Also, authors did not find statistically significant correlation between higher BMI and UUI. In parallel with this, Khullar et al. (12) investigated the incidence of $\mathrm{UI}$ in 13.178 women and they found a significantly higher SUI rate in the obese patients. Similarly, UI, SUI and MUI were statistically more common in poorly educated obese women in the present study ( $p=0.01$ for each parameter).

Previous reports found a significant positive correlation between diabetes mellitus and UI, especially UUI subtype. Furukawa et al. (13) claimed that peripheral neuropathy and micro-vascular damage resulted in UUI. According to Danforth et al. (14) incidence of UUI up to was seen in $82 \%$ of diabetic patients. In another study by Brown et al. (15), prevalence of UUI was significantly higher in diabetic patients and even patients with impaired fasting glucose. In our study, we found a statistically higher diabetes mellitus rate in poorly educated females with UUI and MUI in univariate analysis, however, multivariable regression analysis showed no effect of diabetes mellitus on the development of UUI and MUI ( $p=0.09$ and $p=0.78$ ).

Squamous cells in lower urinary system had an estrogen receptor and deficiency of estrogen in menopausal status has a role in the development of UI with increasing mucosal atrophy in urinary system and total collagen concentration. Zhu et al. (16) reported that the incidence of MUI increased with advancing age and menopausal status. Moreover, according to British cohort study, women in postmenopausal period were reported to have said that their UI complaints were less than pre and perimenopausal women (17). In another study, Rekers et al. (18) reported that MUI was twice more common in postmenopausal women when compared to the ones with premenopausal status. Also, a significant correlation between MUI and postmenopausal status was found in univariate analysis while multivariable regression analysis showed no affect of menopausal status on MUI for poorly educated female population in our study.

What's more, pelvic floor muscle function has a crucial role in preventing $\mathrm{UI}$ and it's well known that pelvic traumas such as pelvic surgeries, deliveries, abortus and difficult labor are related to pelvic floor muscle dysfunction. Wu et al. (19) reported that abortus history and number of cesarean sections were predictive factors for $\mathrm{UI}$ in women aged 20 years and older. In another study, Onur et al. (20) found that presence of difficult labor 
history increased the UI development 1.57 times. In present study we were faced with significantly higher abortus numbers in MUI and difficult labor in women with UUI.

Pelvic organ prolapses and UI, especially SUI subtype, are commonly diagnosed together. Obesity, increased abdominal pressure, lack of hormonal supplement and urogenital trauma may contribute to the development of pelvic organ prolapse and SUI. Islam et al. (21) investigated the predictive factors for UI in women and they found that pelvic organ prolapse was related to MIU (AOR 3.40, 95\% Cl 2.00-5.80; $p<0.0001$ ), and SUI (AOR 2.46, 95\% Cl 1.34-4.52; $p=0.004)$. In parallel, Okonkwo et al. (22) analyzed incontinence status of 3963 women and they found a strong correlation between $\mathrm{UI}$ and pelvic organ prolapse. Also, in present study, multivariable regression analysis revealed that pelvic organ prolapse was significantly related to SUI, UUI and MUI ( $p=0.01$ for each parameter).

The present study had some limitations, though. Cross sectional nature of study without follow up results and absence of treatment outcomes were accepted as limitations in our study. Secondly, period of UI was not examined. Additionally, participants were evaluated by different physicians in three different academic centers. However, to achieve higher internal validity, all physicians were made to undergo the very same education program before the study had started.

\section{CONCLUSION}

All in all, our study revealed that the incidence of UI was very high in poor educated female population and higher BMI, presence of difficult labor, pelvic prolapse were risk factors for UI and UUI. Presence of DM and pelvic organ prolapse were the risk factors for SUI. Moreover, higher BMI, abortus numbers and pelvic organ prolapse were significantly related to MUI. The results of our study, though, must be supported by further randomized studies with larger patient volumes and a prospective nature.

\section{Ethics}

Ethics Committee Approval: Haseki Training and Research Hospital Ethics Committee (4532771-432).

Informed Consent: Form was obtained from the patients.

Peer-review: Externally peer-reviewed.

\section{Authorship Contributions}

Concept: B.Y.Ö., Design: F.Ö., Data Collection or Processing: M.E., S..B.I. M.B., Ö.S., Analysis or Interpretation: B.Y.Ö., F.Ö., Ö.S., U.C.., Literature Search: S.B.I., U.Ç., M.B., Writing: B.Y.Ö., F.Ö.
Conflict of Interest: No conflict of interest was declared by the authors.

Financial Disclosure: The authors declared that this study received no financial support.

\section{REFERENCES}

1. Gajewski JB, Schurch, B, Hamid R, Averbeck M, Sakakibara R, Agrò EF, et al. An International Continence Society (ICS) report on the terminology for adult neurogenic lower urinary tract dysfunction (ANLUTD). Neurourol Urodyn 2018;37:1152-61.

2. Zaccardi JE, Wilson L, Mokrzycki ML. The effect of pelvic floor reeducation on comfort in women having surgery for stress urinary incontinence. Urol Nurs 2010;30:137-46.

3. Hunskaar S, Arnold EP, Burgio K, Diokno AC, Herzog AR, Mallett VT. Epidemiology and natural histology of urinary incontinence. Int Urogynecol J 2000;11:301-19.

4. Pedro J, Brandão T, Schmidt L, Costa ME, Martins MV. What do people know about fertility? A systematic review on fertility awareness and its associated factors. Ups J Med Sci 2018;123:71-81.

5. Ramírez-Moreno JM, Alonso-González R, Peral-Pacheco D, Millán-Núñez MV, Aguirre-Sánchez JJ. Stroke awareness is worse among the old and poorly educated: a population-based survey. J Stroke Cerebrovasc Dis 2015;24:1038-46.

6. Kubik K, Blackwell L, Heit M. Does socioeconomic status explain racial differences in urinary incontinence knowledge? Am J Obstet Gynecol 2004;191:188-93.

7. Simsek A, Ozgor F, Yuksel B, Kucuktopcu O, Kirecci SL, Toptas M, et al. Female sexual function after transobturator tape in women with urodynamic stress urinary incontinence. Springer Plus 2014;3:570.

8. Haylen BT, de Ridder D, Freeman RM, Swift SE, Berghmans B, Lee J, et al. An International Urogynecological Association (IUGA)/International Continence Society (ICS) joint report on the terminology for female pelvic floor dysfunction. Neurourol Urodyn 2010;29:4-20.

9. Stephan E, Hajjar RR. Urinary incontinence in older adults. J Med Liban 2012;60:220-7.

10. Othman JA, Åkervall S, Milsom I, Gyhagen M. Urinary incontinence in nulliparous women aged 25-64 years: a national survey. Am J Obstet Gynecol 2017;216:149.

11. Mishra GD, Hardy R, Cardozo L, Kuh D. Body weight through adult life and risk of urinary incontinence in middle-aged women: results from a British prospective cohort. Int J Obes (Lond) 2008;32:141522.

12. Khullar V, Sexton CC, Thompson CL, Milsom I, Bitoun CE, Coyne KS. The relationship between BMI and urinary incontinence subgroups: results from EpiLUTS. Neurourol Urodyn 2014;33:392-9.

13. Furukawa S, Sakai T, Niiya T, Miyaoka H, Miyake T, Yamamoto S, Kanzaki $S$, et al. Macrovascular Complications and prevalence of urgency incontinence in Japanese patients with type 2 diabetes mellitus: The Dogo Study. Intern Med 2017;56:889-93.

14. Danforth KN, Townsend MK, Curhan GC, Resnick NM, Grodstein F. Type 2 diabetes mellitus and risk of stress, urge and mixed urinary incontinence. J Urol 2009;181:193-7.

15. Brown JS, Vittinghoff E, Lin F, Nyberg LM, Kusek JW, Kanaya AM Prevalence and risk factors for urinary incontinence in women with type 2 diabetes and impaired fasting glucose: findings from the 
National Health and Nutrition Examination Survey (NHANES) 20012002. Diabetes Care 2006;29:1307-12.

16. Zhu L, Lang J, Liu C, Han S, Huang J, Li X. The epidemiological study of women with urinary incontinence and risk factors for stress urinary incontinence in China. Menopause 2009;16:831-6.

17. Kuh D, Cardozo L, Hardy R. Urinary incontinence in middle aged women: childhood enuresis and other lifetime risk factors in a British prospective cohort. J Epidemiol Community Health 1999;53:453-8.

18. Rekers H, Drogendijk AC, Valkenburg HA, Riphagen F. The menopause, urinary incontinence and other symptoms of the genito-urinary tract. Maturitas 1992;15:101-11.
19. Wu XH, Liu XX, Xie KH, Wang RM, Wu YX, Liu YG. Prevalence and related factors of urinary incontinence among Hebei women of China. Gynecol Obstet Invest 2011;71:262-7.

20. Onur R, Deveci SE, Rahman S, Sevindik F, Acik Y. Prevalence and risk factors of female urinary incontinence in eastern Turkey. Int J Urol 2009;16:566-9.

21. Islam RM, Bell RJ, Hossain MB, Davis SR. Types of urinary incontinence in Bangladeshi women at midlife: Prevalence and risk factors. Maturitas 2018;116:18-23.

22. Okonkwo JE, Obionu CO, Obiechina NJ. Factors contributing to urinary incontinence and pelvic prolapse in Nigeria. Int J Gynecol Obstet 2001;74:301-3. 